\title{
Why is Elective Ophthalmic Surgery Cancelled? A Retrospective Evaluation
}

\author{
Mahfouth Bamashmus FRCSEd, FRCOphth ${ }^{1,2^{*}}$, Mohamed Al-Qubaty MBBS, MD ${ }^{3}$, Fady \\ Mukred MBBS, MSc ${ }^{2}$, Abdalsamad Abdalrab MBBS, MSc ${ }^{3}$ and Ezia Alsarory MBBS, MSc ${ }^{3}$
}

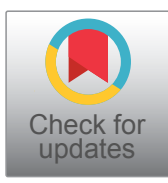

${ }^{1}$ Eye Department, Faculty of Medicine and Health Sciences, Sana'a University, Sana'a, Republic of Yemen, Yemen

${ }^{2}$ Anterior Segment Unit, Magrabi Eye Hospital, Sana'a, Republic of Yemen, Yemen

${ }^{3}$ Anesthesia Department, Magrabi Eye Hospital, Sana'a, Republic of Yemen, Yemen

\begin{abstract}
Aim: Making efficient use of operating rooms (OR) is an important issue so decreasing the cancellation rate of surgery is one method that could increase efficiency. This study was done to report potentially preventable causes of elective ophthalmic surgery cancellations and we reviewed all OR cancellations in 2017 to survey the cancellation causes.

Method: A retrospective review of elective ophthalmic surgery cancellation records and patient medical records was conducted in Yemen Magrabi Eye hospital in Sana'a. Data were collected from the Anesthesiology Quality Assurance Database. We analyzed medical records for cancellations from January 1 to December 31, 2017. Data were analyzed using SPSS 21.0, employing descriptive measures and logistic regression.

Result: One hundred and forty four ophthalmic surgeries were cancelled on the day of surgery at the operating room. This accounted for $1.4 \%$ of total elective ophthalmic surgeries done in the same period. Cases that did not appear or cases that were cancelled at the reception were not included. Cancellation rates varied by patient age, with the rate among the age group $41-60$ being highest (36.8\%) and that among older patients (age $80+$ ) lowest (2.8\%). Of all causes, the majority were considered "preventable", and can be avoided by proper pre-operative evaluation. Forty-eight cases (34\%) were cancelled because of high blood pressure; nineteen cases (13\%) due to high blood sugar and six cases (4\%) due to both high blood pressure and high blood sugar. Chest infection and cough attributed to cancellation of 18 cases $(12 \%)$ and 11 cases were cancelled because patient was not cooperative with local anesthesia. Most operations were done later after the original cancellation issues were addressed and treated.
\end{abstract}

Conclusion: Majority of cancellation causes are avoidable. Medical teams must communicate better with patients and relatives, identify and treat relevant comorbidities, and make adequate preparations for surgery.

\section{Keywords}

Ophthalmic surgery, Anesthesia, Cancellation, Yemen

\section{Introduction}

Making efficient use of operating rooms (OR) is an important issue in decreasing the cancellation rate of surgery and is a method that increases efficiency. Proper preoperative evaluation is the most important factor in decreasing ophthalmic surgery cancellation [1]. Usually the pre-operative evaluation is done by a qualified physician and sometimes refers patients for further evaluation if needed [2]. Almost all ophthalmic surgeries are done as one-day surgery and majority are done under local anesthesia. Elective surgical case cancellation refers to any elective surgical case that is the list on the day prior to surgery but not operated upon as scheduled.

Magrabi Eye Hospital in Sana'a, Republic of Yemen is a tertiary eye hospital that provides eye services not only to the people in the capital city of Sana'a but to people all over Yemen, providing all ophthalmic subspecialties services. A large number of our patients are referred from different governorates due to the paucity of ophthalmic treating facilities in the

*Corresponding author: Mahfouth A Bamashmus FRCSEd FRCOphth, Professor of Ophthalmology, Faculty of Medicine and Health Sciences, Sana'a University, P.O. Box 19576, Sana'a, Republic of Yemen, Yemen, Fax: +967-1210021

Accepted: November 10, 2020

Published online: November 12, 2020

Citation: Bamashmus M, Al-Qubaty M, Mukred F, et al. (2020) Why is Elective Ophthalmic Surgery Cancelled? A Retrospective Evaluation. J Ophthalmic Res Ocular Care 3(1):55-59 
Citation: Bamashmus M, Al-Qubaty M, Mukred F, et al. (2020) Why is Elective Ophthalmic Surgery Cancelled? A Retrospective Evaluation. J Ophthalmic Res Ocular Care 3(1):55-59

country. We observed small number of patients suffer from postponing their elective ophthalmic surgery on the day of surgery. Any elective ophthalmic surgical procedure can be postponed sometimes due to uncontrolled medical problems and sometimes due to simple avoidable reasons. The most common ophthalmic surgical procedure is cataract surgery and the reasons for cancellation in Yemen were discussed in two previous papers [3,4].

Investment on optimal use of operating theatre time is usually an important task for eye hospitals to benefit from theatre staff and surgeons time [5,6]. Ophthalmic surgery cancellation is not well studied and is a reason for waste of resources. Cancellation of elective ophthalmic surgery is a major factor of inefficient use of operating-room time [7]. The operating room is the financial hub of any hospital, and maximizing operating room efficiency has important implications for cost savings, patient satisfaction, and medical team morale [8]. Cancelling a surgery is distressing to the patients and their relatives and usually has direct family issues. In Sana'a city, where the study was conducted, many of our patients come from neighboring and remote governorates; cancellation of an elective surgery, and therefore prolonging patients' stay in Sana'a, is financially and psychologically disturbing for them.

This study was done to report and analyze potentially preventable causes of elective ophthalmic surgery cancellations and we reviewed all operating room cancellations in 2017 to survey the cancellation causes. The data were collected from the hospital records including the daily operating theatre list and the reason for the cancellation.

\section{Material and Method}

A retrospective review of elective ophthalmic surgery cancellation records and patient medical records was conducted in Yemen Magrabi Eye hospital in Sana'a. Magrabi hospital is a tertiary eye hospital providing all subspecialties in ophthalmology.

We analyzed medical records for cancellations from January 1 to December 31, 2017. All patients in the study period and selected for elective ophthalmic surgery and were cancelled due to any reason were included in the study. Data were collected from the Anesthesiology Quality Assurance Database and medical records were reviewed. The operating theatre list is usually generated at 17:00 PM the previous day and includes the patient and surgeons details, the intended ophthalmic surgery and if is done under local or general anesthesia.

The hospital operating rooms open six days a week from 8:00 AM to 3:30 PM using four operating tables. The patient is usually assessed preoperatively by a qualified physician and all required investigations and medications recorded. Preoperative investigations including $\mathrm{CBC}$, random blood sugar, coagulation profile, urea and electrolytes, viral serology (Hepatitis B, Hepatitis C and HIV), IOL calculation and their medical condition assessed for chronic diseases.

Patients that need further evaluation by a cardiologist or endocrinologist are usually referred for evaluation. The anes- thetist reviews the file just before the operation and induction of local or general anesthesia.

Patients included in this study were those who were scheduled for elective ophthalmic surgery and were not done on that day. Cases that did not appear or cases that were cancelled at the reception were not included. Emergency cases were excluded. At the end of the day the anesthetist and the chief theatre staff fill a form of the cases that were cancelled and the reason for the cancellation.

Data including the cancellation reason were entered into a database using and Excel 2010 programme were analyzed using SPSS 21.0, employing descriptive measures. Information regarding the patient age, gender and reason for cancellation were recorded. The decision for cancellation is usually taken by the ophthalmologist, the anesthetist or the patients and their relatives.

Table 1 shows reasons of elective ophthalmic surgery cancellation in Magrabi Eye Hospital, Sana'a. Table 2 shows cancellation according to elective ophthalmic surgical procedure. As the study was considered a quality assurance project by the Research Ethics Committee of Magrabi Eye Hospital, it did not require ethical approval.

\section{Results}

One hundred and forty four elective ophthalmic surgeries were cancelled on the day of surgery at the operating room. Females $(81 / 144 ; 56.2 \%)$ were cancelled more than males (63/144; 43.8\%). Age was ranging from three months to $80+$ years. Cancellation rates varied by patient age, $0-17$ years $(9$; $6.3 \%), 18-40$ years $(42 ; 29.2 \%), 41-60(53 ; 36.8 \%), 61-80$ years (36; $25.0 \%)$ and age $80+(4 ; 2.7 \%)$.

A total of 10,350 patients were planned and listed for elective ophthalmic surgery over the 12 months period in 2017. Cases that were postponed and cancelled were studied and the reason for surgery cancellation was analyzed. One hundred and forty four cases were cancelled (1.4\%) due to different reasons.

More decisions for postponement came from anesthetist. The anesthetist cancelled (98; 68.1\%), the surgeon (ophthalmologist) cancelled $(27 ; 18.8 \%)$, the patients or relatives were responsible for $(19 ; 13.1 \%)$ of cancelled cases (Table 1$)$.

Of the total causes, majority was considered "preventable", and can be avoided by proper pre-operative evaluation. Forty-eight cases (33.3\%) were cancelled because of high blood pressure; nineteen cases due to high blood sugar (13.2\%) and six cases (4.2\%) due to both high blood pressure and high blood sugar. Chest infection and cough attributed to cancellation of 18 cases (12.5\%) and 11 cases (7.6\%) were cancelled because patient was not cooperative with local anesthesia (Table 1).

The most common elective ophthalmic surgery that was cancelled was phacoemulsification with intraocular lens implantation followed by pars plana vitrectomy with silicone oil injection (Table 2). Most operations were done later after the original cancellation issues were addressed and treated. 
Citation: Bamashmus M, Al-Qubaty M, Mukred F, et al. (2020) Why is Elective Ophthalmic Surgery Cancelled? A Retrospective Evaluation. J Ophthalmic Res Ocular Care 3(1):55-59

Table 1: Reasons of elective ophthalmic surgery cancellation in Magrabi Eye Hospital, Sana'a.

\begin{tabular}{|c|c|c|c|}
\hline Cancellation decision maker & Reason of cancellation & No of patients & $\%$ \\
\hline \multirow{7}{*}{$\begin{array}{l}\text { Anesthetist } \\
(98,68.1 \%)\end{array}$} & Uncontrolled Systemic Hypertension & 48 & 33.3 \\
\hline & Uncontrolled Diabetes Mellitus (DM) & 19 & 13.2 \\
\hline & Chest Infection and Cough & 18 & 12.5 \\
\hline & Uncontrolled Systemic Hypertension \& DM & 6 & 4.2 \\
\hline & Cardiac problems and need cardiac consultation & 3 & 2.1 \\
\hline & Difficult intubation & 2 & 1.4 \\
\hline & Not fit for general anesthesia & 2 & 1.4 \\
\hline \multirow{7}{*}{$\begin{array}{l}\text { Ophthalmologist } \\
(27,18.8 \%)\end{array}$} & Uncooperative patients for local anesthesia & 11 & 7.6 \\
\hline & Eye Infections (conjunctivitis or blepharitis) & 4 & 2.8 \\
\hline & Watery eyes that needs DCR & 4 & 2.8 \\
\hline & Need ENT consultation & 3 & 2.1 \\
\hline & Facial \& hand abscess & 2 & 1.4 \\
\hline & High intraocular pressure after local anesthesia & 2 & 1.4 \\
\hline & Power of intraocular lens not available & 1 & 0.7 \\
\hline \multirow{6}{*}{$\begin{array}{l}\text { Patient-related factors } \\
(19,13.1 \%)\end{array}$} & Patient not fasting for general anesthesia & 5 & 3.4 \\
\hline & Patient or relative do not want surgery & 5 & 3.4 \\
\hline & Did not stop aspirin & 4 & 2.8 \\
\hline & Patient found to be pregnant & 2 & 1.4 \\
\hline & $\begin{array}{l}\text { Patient have diarrhea, postpartum bleeding, urinary tract } \\
\text { infection }\end{array}$ & 3 & 2.1 \\
\hline & Total & 144 & 100 \\
\hline
\end{tabular}

DCR: Dacryocystorhinostomy; ENT: Ear, Nose and Throat.

Table 2: Cancellation according to ophthalmic surgical procedure.

\begin{tabular}{|l|l|l|}
\hline Name of ophthalmic surgical procedure & Number of cases cancelled & \% \\
\hline Phacoemulsification + intraocular lens implantation (IOL) & 48 & 33.3 \\
\hline Pars plana vitrectomy + silicone oil injection & 36 & 25.0 \\
\hline Dacryocystorhinostomy (DCR) & 15 & 10.3 \\
\hline Pterygium excision & 6 & 4.2 \\
\hline Trabeculectomy & 6 & 4.2 \\
\hline Evisceration or enucleation & 6 & 2.2 \\
\hline Squint surgery & 4 & 2.1 \\
\hline Phakic intraocular lens (ICL) & 3 & 2.1 \\
\hline Avastin intravitreous injection & 3 & 2.1 \\
\hline Lensectomy + anterior vitrectomy & 3 & 2.1 \\
\hline Phaco-trabeculectomy + intraocular lens implantation & 3 & 2.1 \\
\hline Extracapsular cataract surgery + IOL & 3 & 1.4 \\
\hline Lid skin graft & 2 & 1.4 \\
\hline Secondary porous implantation & 2 \\
\hline Silicone oil removal & 2 & 1.4 \\
\hline Crosslinking for keratoconus & 1 & 0.7 \\
\hline Chalazion removal & 1 & 0.7 \\
\hline Total & 144 \\
\hline
\end{tabular}


Citation: Bamashmus M, Al-Qubaty M, Mukred F, et al. (2020) Why is Elective Ophthalmic Surgery Cancelled? A Retrospective Evaluation. J Ophthalmic Res Ocular Care 3(1):55-59

\section{Discussion}

Most causes of cancellation of elective ophthalmic surgeries are avoidable. Eye hospitals aim to provide efficient eye services and to do so cancellation rate have to be reduced. A low cancellation rate of any ophthalmic surgery indicates an efficient ophthalmic service $[7,8]$.

Most causes of cancellation of elective ophthalmic surgeries were potentially avoidable $[3,9]$. Medical teams must communicate better with patients and relatives, identify and treat relevant comorbidities, and make adequate preparations for surgery. Achieving this target will reduce the unused operating time on the day of surgery $[2,10]$.

Cancellation of ophthalmic surgery can be due to preventable and non-preventable causes $[3,4,9]$. A study done in Kuwait Hospital in Sana'a concluded that operation theatres do not use their full capacity because of cataract surgery cancellation which reached to $12 \%$ and this increases the waiting lists [3]. Inefficiency of management sometimes is reflected in increase of cancellation of planed ophthalmic surgeries [7]. Usually cancellation causes are classified by different factors such us surgeon, anesthetist, patient and hospital factors.

Ophthalmic surgery cancellation is costly and leads to dissatisfied patients and relatives. Some patients and their relatives can go through emotional trauma because of the loss of time and the need to come back again for the surgery [11].

Anesthesia pre-operative medicine clinic (APMC) has been established in many countries to avoid cancellation on the day of surgery [12]. This clinic is not practical in ophthalmic surgery because majority of cases are done under local anesthesia. It is successful in specialties were majority of cases are done under general anesthesia were this clinic optimizes the medical condition of the patient before surgery and anesthesia administration [13]. These clinics have enhanced patient satisfaction and safety and reduced the duration of staying in the hospital [14]. Pre-operative assessment has evolved in response to more operations and changes in the surgical population. Patients are older and have more long-term medical comorbidities. At the same time, there has been a move from paternalistic medical decision-making to shared decision-making, based on an individual patient's choice or preference $[12,13]$.

Magrabi hospital provides subspecialty ophthalmic services and a high number of patients come from different governorates and cancelling their surgery has a high cost rate as majority of them stay in hotels with increase in their daily expenses. The physician who is in charge of the preoperative evaluation needs to ask for opinion of the cardiac, endocrine and anesthetist to decrease postponing ophthalmic surgeries.

Anesthesiologist cancels surgeries because of uncontrolled hypertension or diabetes and cases with chest problems (COPD or asthma). Ophthalmologists usually cancel surgery because of local eye problems namely blepharitis, conjunctivitis, uncooperative patient or high intraocular pressure after local anesthesia. Preoperative evaluation by a trained physician with the support of the anesthetist plays a significant role in decreasing cancellation rates $[12,13]$. Patients who have multiple medical diseases should visit their physician preoperatively.

Cancellation of elective surgical procedures is inconvenient and stressful on patients, causing loss of working days and disruption of daily life. Furthermore, it causes significant emotional trauma to the patients and their families [11]. Parents of children waiting for pediatric general surgery operations thought that the need for the operation was significantly more urgent then their classification of elective [15]. It is important to satisfy patients' expectations to offer a better assistance, in order to ensure a comforting and friendly experience, especially when the patients are weak and not young anymore [11]. When a case is cancelled, money is potentially wasted on unnecessary setups, including sterilization, disposable instruments and sutures $[6,7]$.

\section{Conclusions}

Of all causes of cancellation of surgery, majority of causes are avoidable. Medical teams must communicate better with patients and relatives, identify and treat relevant comorbidities, and make adequate preparations for surgery. Ensuring efficiency in both the clinic and in the operating room is becoming mandatory in eye clinics and hospitals. One factor to improve the efficiency is to minimize cancellations of elective ophthalmic surgeries.

\section{Financial Support}

None.

\section{Proprietary Interest}

None.

\section{References}

1. Henderson BA, Naveiras M, Butler N, et al. (2006) Incidence and causes of ocular surgery cancellations in an ambulatory surgical center. Cataract Refract Surg 32: 95-102.

2. Keay L, Lindsley K, Tielsch J, et al. (2019) Routine preoperative medical testing for cataract surgery. Cochrane Database Syst Rev 1: CD007293.

3. Bamashmus M, Haider T, Al-Kershy R (2010) Why is cataract surgery canceled? A retrospective evaluation. Eur J Ophthalmol 20: 101-105.

4. Al-Shaer M, Al-Akily S, Bamashmus M, et al. (2017) Why is cataract surgery cancelled in eye camps: A prospective evaluation. Sudanese J Ophthalmol 9: 62-66.

5. Levine WC, Dunn PF (2015) Optimizing operating room scheduling. Anesthesiol Clin 33: 697-711.

6. Kahloul M, Nakhli MS, Jebali C, et al. (2019) Assessment of the operating room efficiency by the real time of room occupancy. Tunis Med 97: 675-680.

7. Madni TD, Imran JB, Clark AT, et al. (2018) Prospective evaluation of operating room inefficiency. J Burn Care Res 39: 977-981.

8. Rothstein DH, Raval MV (2018) Operating room efficiency. Semin Pediatr Surg 27: 79-85.

9. Shaikh RM, Al-Yafi A, Malak M (2007) Causes of ocular surgery cancellation and the need of anesthesia preoperative medicine 
clinic. Saudi Journal of Ophthalmology 21: 149-152.

10. Mayro EL, Pizzi LT, Hark LA, et al. (2018) A proposed intervention to decrease resident-performed cataract surgery cancellation in a tertiary eye care center. Am Health Drug Benefits 11: 480-487.

11. Dell'Atti $L$ (2014) The cancelling of elective surgical operations causes emotional trauma and a lack of confidence: Study from a urological department. Urologia 81: 242-245.

12. Dhesi JK, Swart M (2016) Specialist pre-operative assessment clinics. Anaesthesia 71: 3-8.
13. Ferschl MB, Tung A, Sweitzer NJ, et al. (2005) Preoperative clinic visits reduce operating room cancellations and delays. Anesthesiology 103: 855-859.

14. Hepner DL, Bader AM, Hurwitz S, et al. (2004) Patient satisfaction with preoperative assessment in a preoperative assessment testing clinic. Anesth Analg 98: 1099-1105.

15. Miller GG (2004) Waiting for an operation: Parent's perspectives. Can J Surg 47: 179-181. 